\title{
A "Universal" Class of 4-Coloured Graphs
}

\author{
M. MULAZZANI
}

\begin{abstract}
A family of 4-coloured graphs depending on three integers $b, l, t$ and on a transitive pair of permutations $\sigma, r \in \Sigma_{b}$ is constructed. Each associated topological space turns out to be a $b$-fold branched covering of either a $\theta$ - or a handcuff-graph, with embedding depending on $l$ and $t$, or a two-bridge knot or link of type $(l, t)$. Moreover, the monodromy map is completely defined by $\sigma$ and $\tau$. In particular, when $l=2$ and $t=1$, the space is homeomorphic to the (possibly singular) manifold $N(\sigma, \tau)$, which is the branched covering of the Montesinos universal graph, associated to the pair $\sigma, \tau$. This allows us to obtain a "universal" class of 4-coloured graphs representing all orientable 3-dimensional singular manifolds. Further, the necessary and sufficient condition for the graph to represent a manifold is obtained and a topological interpretation of a similar construction of $\mathrm{A}$. Cavicchioli is given.
\end{abstract}

1991 Mathematics Subject Classification : Primary 57M25, 57M12; Secondary $57 \mathrm{M} 15$.

Servicio publicaciones Univ. Complutense. Madrid, 1996.

Work performed under the auspices of the GNSAGA-CNR, within the project "Geometria reale e complessa" of the MURST and under the supervision of Prof. Massimo Ferri. 


\section{INTRODUCTION AND NOTATIONS}

Throughout this paper, all spaces and maps are piecewise-linear (PL) in the sense of [24] or [15]; (quasi-) manifolds are always supposed to be closed and connected. The term "graph" will be used instead of "multigraph", hence loops are forbidden but multiple edges are allowed. Graphs will always be of finite type. As a general reference for graph theory see [13] and for the theory of branched coverings [1], [9] and [19].

Now, we recall some necessary notions on edge-coloured graphs, pseudocomplexes and the relations between them (a detailed survey on these subjects can be found in [8]).

An $(n+1)$-coloured graph is a pair $\Omega=(\Gamma, \gamma)$, where $\Gamma=(V(\Gamma)$, $E(\Gamma))$ is a connected regular graph of degree $n+1$ and $\gamma: E(\Gamma) \rightarrow N_{n}=$ $\{0,1,2, \ldots, n\}$ is a map such that $\gamma\left(e^{\prime}\right) \neq \gamma\left(e^{\prime \prime}\right)$, for any two adjacent edges $e^{\prime}, e^{\prime \prime}$. We set $V(\Omega)=V(\Gamma)$ and $E(\Omega)=E(\Gamma)$. An edge $e \in E(\Gamma)$ such that $\gamma(e)=k$ is called a $k$-edge.

An $(n+1)$-coloured graph $\Omega$ naturally induces an $(n+1)$-tuple $\left(\iota_{0}, \iota_{1}, \iota_{2}, \ldots, \iota_{n}\right)$ of fixed-point-free involutions $\iota_{k}: V(\Omega) \rightarrow V(\Omega)$, by setting $\iota_{k}(v)=w$ iff $v$ and $w$ are the vertices of a $k$-coloured edge. Conversely, an $(n+1)$-tuple $\iota=\left(\iota_{0}, \iota_{1}, \iota_{2}, \ldots, \iota_{n}\right)$ of fixed-point-free involutions on a finite set $V$ defines an $(n+1)$-coloured graph $\Omega$ with $V(\Omega)=V$, $E(\Omega)=\left\{\left(\left\{v, \iota_{k}(v)\right\}, k\right) \mid v \in V, k \in N_{n}\right\}$ and $\gamma\left(\left\{v, \iota_{k}(v)\right\}, k\right)=k$. We also denote $\Omega$ by $(V, \iota)$.

A morphism between two $(n+1)$-coloured graphs $\Omega^{\prime}=\left(V^{\prime}, \iota^{\prime}\right)$ and $\Omega=(V, \iota)$ is a pair $(f, \rho)$, where $\rho$ is a permutation on $N_{n}$ and $f: V^{\prime} \rightarrow V$ is a map such that $f \circ \iota_{c}^{\prime}=\iota_{\rho(c)} \circ f$, for every $c \in N_{n}$. A morphism is said to be a $c p$-morphism (coloured preserving) when $\rho=1$ (the identity permutation), hence we shall denote a cp-morphism simply by a map $f$. If $f$ is a bijection, then the (cp-) morphism will be called a (cp-) isomorphism.

For every $\mathcal{F} \subseteq N_{n}$, we call $\mathcal{F}$-residue each connected component of the subgraph $\Omega_{\mathcal{F}}$ having $V(\Omega)$ as vertex set and $\gamma^{-1}(\mathcal{F})$ as edge set. An $m-$ residue is an $\mathcal{F}$-residue such that $\# \mathcal{F}=m$. We can think of an $m$ residue as an $m$-coloured graph, by means of a bijection $\beta: \mathcal{F} \rightarrow N_{m-1}$. Let $f$ be a cp-morphism between $\Omega^{\prime}$ and $\Omega$, and let $\Lambda$ be any $\mathcal{F}$-residue of $\Omega$, then $f^{-1}(V(\Lambda))$ consists of the vertices of a certain set of $\mathcal{F}$-residues 
$\Lambda_{i}^{\prime}$ of $\Omega^{\prime}$. We say that $\Lambda$ is ordinarily covered by $f$ iff $f$ is injective when restricted to the vertices of each $\Lambda_{i}^{\prime}$.

An $n$-dimensional pseudocomplex $K$ is an $n$-dimensional ball-complex in which every $h$-ball, considered with all its faces, is isomorphic with the complex underlying an $h$-simplex (see [14], p. 49). For this reason, each $h$-ball of $K$ is called $h$-simplex. Note that the first derived subdivision $\operatorname{Sd}(K)$ of $K$ is a usual simplicial complex. The $h-$ skeleton of $K$ is defined as in the simplicial case: $S^{h}(K)=\{s \in K \mid \operatorname{dim}(s) \leq h\}$. We can think to a graph $\Gamma$ as a 1-pseudocomplex $\bar{\Gamma}$.

An $n$-pseudocomplex $K$ is said to be homogeneous if every simplex $s \in K$ is a face of an $n$-simplex of $K$. If $H$ is an $n$-homogeneous pseudocomplex, then the disjoined star $\operatorname{Std}(s, H)$ of a simplex $s \in H$ is the subcomplex $\operatorname{St}\left(s^{\prime}, \operatorname{Sd}(H)\right)$ of $\operatorname{Sd}(H)$, where $s^{\prime} \in \operatorname{Sd}(H)$ is any simplex ${ }^{1}$ such that $s^{\prime} \subseteq s$ and $\operatorname{dim}\left(s^{\prime}\right)=\operatorname{dim}(s)$. Furthermore, $H$ is said to be strongly connected if each pair $s^{\prime}, s^{\prime \prime}$ of $n$-simplices of $H$ are connected by a sequence of $n$-simplices $s_{0}, s_{1}, s_{2}, \ldots, s_{m} \in H$ such that $s_{0}=s^{\prime}$, $s_{m}=s^{\prime \prime}$ and the pair of simplices $s_{i-1}, s_{i}$ have a common $(n-1)$-face, for $1 \leq i \leq m$. The underlying polyhedron $|H|$ of an $n$-homogeneous pseudocomplex $H$ is called a quasi-manifold iff (i) the disjoined star of every simplex of $H$ is strongly connected and (ii) each $(n-1)$-simplex is a face of exactly two $n$-simplices [10]. A 3-dimensional quasi-manifold is also called a singular 3-manifold in [18].

We can associate in a standard way an $n$-dimensional pseudocomplex $K(\Omega)$ to an $(n+1)$-coloured graph $\Omega=(V, \iota)$ by the following rules (see [6]):

(1) take a $n$-simplex $\sigma^{n}(x)$ for each $x \in V$, and label its vertices by $N_{n}$;

(2) if $x, y \in V$ and $y=\iota_{c}(x)$, then identify the $(n-1)$-faces of $\sigma^{n}(x)$ and $\sigma^{n}(y)$ opposite to the vertices labeled by $c$, so that equally labeled vertices are identified together.

The graph $\Omega$ represents $K(\Omega)$ and all spaces homeomorphic to $|K(\Omega)|$. Clearly, $|K(\Omega)|$ is connected because $\Omega$ is connected too. As a matter of fact, $|K(\Omega)|$ is precisely a quasi-manifold [10] and it is orientable iff $\Omega$ is bipartite [8]. If two $(n+1)$-coloured graphs $\Omega^{\prime}$ and $\Omega^{\prime \prime}$

${ }^{1}$ The definition does not depend on the choice of $s^{\prime}$, up to isomorphism of pseudocomplexes. 
are isomorphic then $\left|K\left(\Omega^{\prime}\right)\right|$ and $\left|K\left(\Omega^{\prime \prime}\right)\right|$ are obviously homeomorphic. If $\Omega=(V, \iota)$ is an $(n+1)$-coloured graph such that $\iota_{c}=\iota_{c^{\prime}}$, for two different colours $c, c^{\prime} \in N_{n}$, then $|K(\Omega)|$ is homeomorphic to the suspension $\Sigma(X)$ of the $(n-1)$-dimensional space $X=\left|K\left(\Omega_{\mathcal{F}}\right)\right|$, where $\mathcal{F}=N_{n}-\{c\}$.

The construction of $K(\Omega)$ naturally defines a "labelling" map $\Phi$ : $S^{0}(K(\Omega)) \rightarrow N_{n}$ which is injective when restricted to the vertices of any simplex of $K(\Omega)$. Moreover, there is a bijection $\Psi$ between the set of all residues of $\Omega$ and the set of all simplices of $K(\Omega)$ (including the empty simplex), sending each $\mathcal{F}$-residue to an $(n-\# \mathcal{F})$-simplex whose vertices are labelled by $N_{n}-\mathcal{F}$. This bijection reverses the inclusions. So, when $\Omega=(\Gamma, \gamma)$ represents a manifold, the 1-pseudocomplex $\bar{\Gamma}$ is isomorphic to the 1-skeleton of the ball-complex dual to $K(\Omega)$. For the 3-dimensional case, the singular set of $|K(\Omega)|$ consists of a finite number of points [3]. Each singular point corresponds, via $\Psi$, to a 3 -residue of $\Omega$ not representing $S^{2}$.

An $(n+1)$-coloured graph which represents a manifold is called an $n$-gem; moreover an $n$-gem with exactly $n+1 n$-residues is called a crystallization. Every manifold admits representations by gems [16] and by crystallizations [23]. All 2- and 3-coloured graphs are gems and, since the underlying polyhedron of a 3-dimensional pseudocomplex $K$ is a manifold iff its Euler characteristic vanishes ([25], p. 216), a 4-coloured graph represents a 3-manifold iff $\alpha_{2}-\alpha_{0}-\alpha_{3}=0$, where $\alpha_{0}, \alpha_{2}, \alpha_{3}$ are respectively the number of 0 -residues (vertices), 2 -residues (bicoloured cycles) and 3-residues of the graph [16]. For higher dimensions the problem of characterizing $n$-gems among $(n+1)$-coloured graphs is still open .

A 2-cell embedding of an $(n+1)$-coloured graph $\Omega$ into a surface is said to be regular iff there exists a permutation $\xi$ on $N_{n}$, which is a cycle of order $n+1$ and such that each region of the embedding has boundary composed by a $\{c, \xi(c)\}$-residue, for a suitable $c \in N_{n}$ (see [11]). If an $(n+1)$-coloured graph $\Omega$ regularly embeds in $S^{2}$, then the space $|K(\Omega)|$ is homeomorphic to $S^{n}$ (see [7]).

Let $\Omega^{\prime}=\left(V^{\prime}, \iota^{\prime}\right)$ and $\Omega=(V, \iota)$ be $(n+1)$-coloured graphs. A cp-morphism $f$ between two $(n+1)$-coloured graphs $\Omega^{\prime}=\left(V^{\prime}, \iota^{\prime}\right)$ and $\Omega=(V, b)$ is said to be a 1-covering [27] if there exists a 2-residue of $\Omega$ not ordinarily covered by $f$. A 1-covering naturally induces a PL-map $|f|:\left|K\left(\Omega^{\prime}\right)\right| \rightarrow|K(\Omega)|$, which is a $\left(\frac{\# V\left(\Omega^{\prime}\right)}{\# V(\Omega)}\right)$-fold branched covering 
map. If $\Omega$ represents a manifold, then the branching set consists of the homogeneous $(n-2)$-subcomplex of $K(\Omega)$, whose $(n-2)$-simplices are associated, via $\Psi$, to the 2 -residues of $\Omega$ not ordinarily covered by $f$ (see [17]).

\section{CONSTRUCTION OF THE FAMLY}

Let $b, l, t$ be parameters such that $b, l \in \mathbb{Z}^{+}, t \in \mathbb{Z}_{2 l}$ and $\operatorname{gcd}(l, t)=$ 1. Further, let $\sigma, \tau \in \Sigma_{b}$ be a transitive pair of permutations on $\mathbb{Z}_{b}$. We consider the 4-coloured graph $G_{m}(b, l, t, \sigma, \tau)$ having as vertex set

$$
V\left(G_{m}(b, l, t, \sigma, \tau)\right)=\mathbb{Z}_{b} \times \mathbb{Z}_{2 l}
$$

and equipped with the four fixed-point-free involutions:

$$
\begin{aligned}
& \iota_{0}(i, j)=\left(i, j-(-1)^{j}\right), \\
& \iota_{1}(i, j)=\left(i, j+(-1)^{j}\right), \\
& \iota_{2}(i, j)=\left(\sigma^{\eta(j)}(i), 1-j\right), \\
& \iota_{3}(i, j)=\left(\tau^{-\eta(j-t)}(i), 1-j+2 t\right),
\end{aligned}
$$

where $\eta: Z_{2 l} \rightarrow\{-1,+1\}$ is the function

$$
\eta(j)=\left\{\begin{array}{ll}
+1 & \text { if } 1 \leq j \leq l \\
-1 & \text { otherwise }
\end{array} .\right.
$$

The graph is bipartite and connected, therefore its associated space $S_{m}(b, l, t, \sigma, \tau)$ is a 3 -dimensional orientable quasi-manifold. In particular, the family $\mathcal{G}_{m}=\left\{G_{m}(b, l, t, \sigma, \tau)\right\}$ contains the Lins-Mandel family $\mathcal{G}=\left\{G(b, l, t, c) \mid c \in \mathbb{Z}_{b}\right\}[16]$, the Lins-Mandel extended family $\tilde{\mathcal{G}}=\left\{\tilde{G}\left(b, l, t, c, c^{\prime}\right) \mid c, c^{\prime} \in \mathbb{Z}_{b}, \operatorname{gcd}\left(b, c, c^{\prime}\right)=1\right)[21]$ and the Cavicchioli family $\mathcal{G}_{a}=\left\{G_{a}(b, l, t, \alpha) \mid \alpha \in \Sigma_{b}\right.$ is a cycle of order $\left.b\right\}$ [4], as stated in the following:

Lemma 2.1, Let $v=\left(\begin{array}{llll}0 & 1 & 2 & \ldots\end{array}\right.$
(a) $G(b, l, t, c)=G_{m}\left(b, l, t, v, v^{-c}\right)$;
(b) $\tilde{G}\left(b, l, t, c, c^{\prime}\right)=G_{m}\left(b, l, t, v^{c^{\prime}}, v^{-c}\right)$;
(c) $G_{a}(b, l, t, \alpha)=G_{m}\left(b, l, t, v, \alpha^{-1}\right)$. 
Proof. Observe first that $G(b, l, t, c), \tilde{G}\left(b, l, t, c, c^{\prime}\right)$ and $G_{a}(b, l, t, \alpha)$ have the same vertex set of $G_{m}(b, l, t, \sigma, \tau)$. Let now $\left(\iota_{0}^{\prime}, \iota_{1}^{\prime}, \iota_{2}^{\prime}, \iota_{3}^{\prime}\right),\left(\iota_{0}^{\prime \prime}, \iota_{1}^{\prime \prime}\right.$, $\left.\iota_{2}^{\prime \prime}, \iota_{3}^{\prime \prime}\right)$ and $\left(\iota_{0}^{\prime \prime \prime}, \iota_{1}^{\prime \prime \prime}, \iota_{2}^{\prime \prime \prime}, \iota_{3}^{\prime \prime \prime}\right)$ be the involutions defining the three graphs respectively (see [16], [21] and [4]), then we get:

(a) $\iota_{0}^{\prime}=\iota_{0}, \iota_{1}^{\prime}=\iota_{1}, \iota_{2}^{\prime}(i, j)=(i+\eta(j), 1-j)=\left(v^{\eta(j)}(i), 1-j\right)$ and $\iota_{3}^{\prime}(i, j)=(i+c \eta(j-t), 1-j+2 t)=\left(v^{c \eta(j-t)}(i), 1-j+2 t\right)$;

(b) $\iota_{0}^{\prime \prime}=\iota_{0}, \iota_{1}^{\prime \prime}=\iota_{1}, \iota_{2}^{\prime \prime}(i, j)=\left(i+c^{\prime} \eta(j), 1-j\right)=\left(v^{c^{\prime} \eta(j)}(i), 1-j\right)$ and $\iota_{3}^{\prime \prime}=\iota_{3}^{\prime}$;

(c) $\iota_{0}^{\prime \prime \prime}=\iota_{0}, \iota_{1}^{\prime \prime \prime}=\iota_{1}, \iota_{2}^{\prime \prime \prime}=\iota_{2}^{\prime}$ and $\iota_{3}^{\prime \prime \prime}(i, j)=\left(\alpha^{\eta(j-t)}(i), 1-j+2 t\right)$.

Let $\mathcal{S}_{m}$ be the family of spaces $\left\{S_{m}(b, l, t, \sigma, \tau) \mid G_{m}(b, l, t, \sigma, \tau) \in\right.$ $\left.\mathcal{G}_{m}\right\}$. Next lemma will give some homeomorphisms among elements of $\mathcal{S}_{m}$.

\section{Lemma 2.2.}

(a) The graphs $G_{m}(b, l, t, \sigma, \tau), G_{m}(b, l, t, \tau, \sigma)$, and $G_{m}\left(b, l, t, \sigma^{-1}, \tau^{-1}\right)$ are isomorphic; thus, $S_{m}(b, l, t, \sigma, \tau) \cong S_{m}(b, l, t, \tau, \sigma) \cong S_{m}(b, l, t$, $\left.\sigma^{-1}, \tau^{-1}\right)$.

(b) $G_{m}(b, l, t, \sigma, \tau)$ is equal to $G_{m}\left(b, l, l+t, \sigma, \tau^{-1}\right)$ and isomorphic to $G_{m}\left(b, l, l-t, \sigma^{-1}, \tau\right) ;$ thus, $S_{m}(b, l, t, \sigma, \tau) \cong S_{m}\left(b, l, l+t, \sigma, \tau^{-1}\right) \cong$ $S_{m}\left(b, l, l-t, \sigma^{-1}, \tau\right)$

(c) If $\tau=1$ (resp. $\sigma=1$ ), then $\sigma$ (resp. $\tau$ ) is a cycle of order $b$ and $S_{m}(b, l, t, \sigma, \tau) \cong S^{3}$.

(d) If $S_{m}(b, 1,1, \sigma, \tau)$ is a manifold, then it is homeomorphic to $S^{3}$.

Proof. (a) Let $\phi$ be the bijection on $\mathbb{Z}_{b} \times \mathbb{Z}_{2 l}$ defined by $\phi(i, j)=$ $(i, 1-j+t)$. Then, when $t$ is even ( resp. when $t$ is odd), an isomorphism (see Section 1 ) between $G_{m}(b, l, t, \sigma, \tau)$ and $G_{m}(b, l, t, \tau, \sigma)$ is given by the pair $(\phi,(23))$ (resp. by the pair $(\phi,(01)(23)))$. Let $\phi(i, j)=(i, j+l)$. If $l$ is odd (resp. even), then $\left(\phi,\left(\begin{array}{l}0 \\ 1\end{array}\right)\right)$ is an isomorphism (resp. $\phi$ is a cp-isomorphism) between $G_{m}(b, l, t, \sigma, \tau)$ and $G_{m}\left(b, l, t, \sigma^{-1}, \tau^{-1}\right)$. 


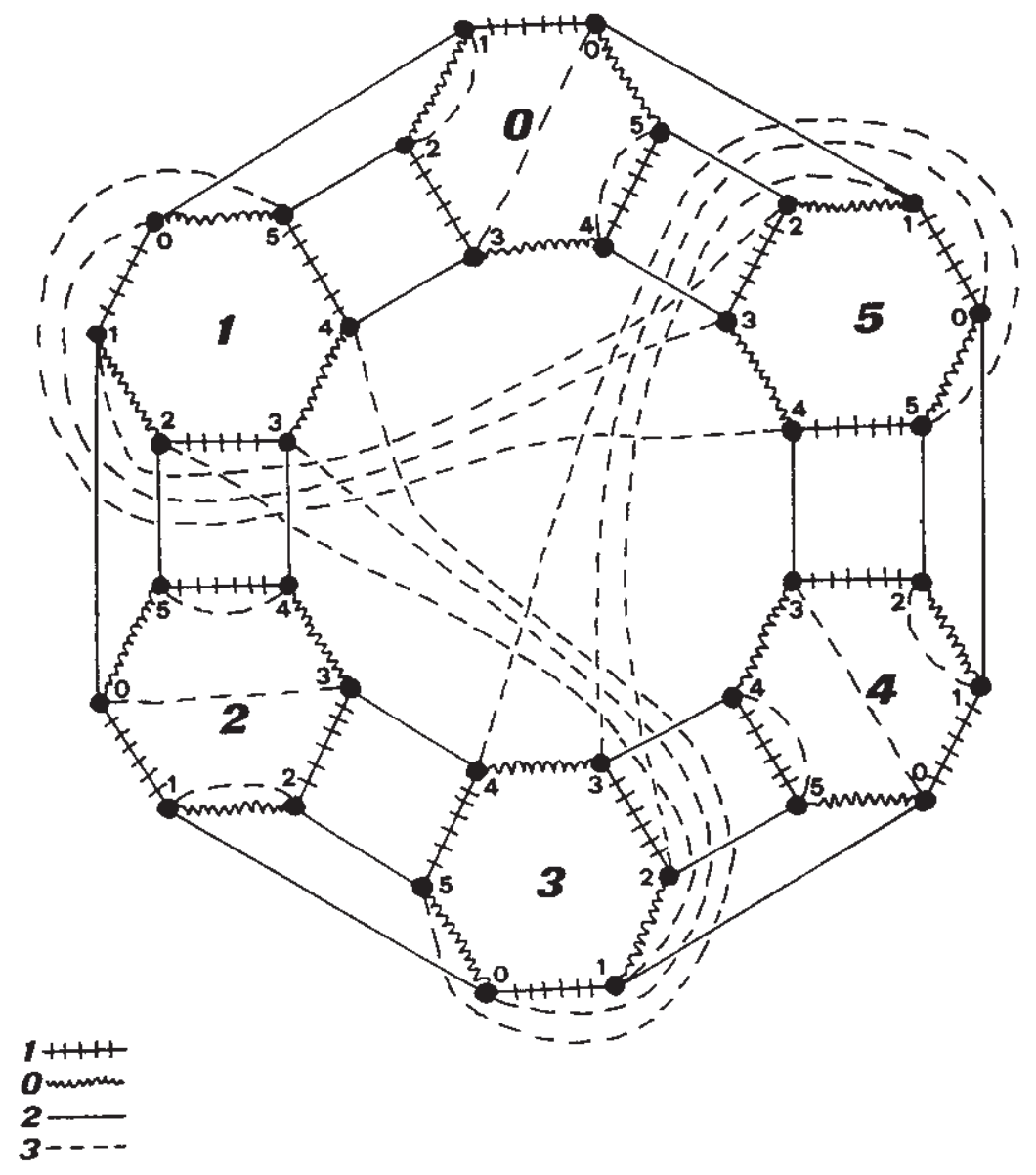

Figure 1. $G_{m}\left(6,3,1,\left(\begin{array}{lllll}0 & 1 & 2 & 3 & 4\end{array}\right),\left(\begin{array}{lll}1 & 5 & 3\end{array}\right)\right)$ 
(b) Let $\left(\iota_{0}^{\prime}, \iota_{1}^{\prime}, \iota_{3}^{\prime}, \iota_{3}^{\prime}\right)$ be the 4 -tuple of involutions associated to $G_{m}\left(b, l, l+t, \sigma, \tau^{-1}\right)$. Clearly, $\iota_{0}^{\prime}=\iota_{0}, \iota_{1}^{\prime}=\iota_{1}, \iota_{2}^{\prime}=\iota_{2}$ and, since $\eta(j-l)=-\eta(j)$ for all $j \in \mathbb{Z}_{2 l}, \iota_{3}^{\prime}(i, j)=\left(\tau^{\eta(j-l-t)}(i), 1-j+2 l+\right.$ $2 t)=\left(\tau^{-\eta(j-t)}(i), 1-j+2 t\right)=\iota_{3}(i, j)$. Therefore, $G_{m}(b, l, t, \sigma, \tau)=$ $G_{m}\left(b, l, l+t, \sigma, \tau^{-1}\right)$. Finally, let $\phi(i, j)=(i, 1-j)$, then $\phi$ is a cpisomorphism between $G_{m}(b, l, t, \sigma, \tau)$ and $G_{m}\left(b, l, l-t, \sigma^{-1}, \tau\right)$.

(c) The results of point (a) allow us to prove only the case $\tau=1$. Since $(\sigma, \tau)$ is a transitive permutation pair, $\sigma$ must generate a transitive subgroup of $\Sigma_{b}$ and therefore is a cycle of order $b$. Up to equivalence, we can suppose $\sigma=v=\left(\begin{array}{llll}0 & 1 & 2 & \cdots\end{array}-1\right)$. It is easy to find a planar regular embedding of $G_{m}(b, l, t, v, 1)$ (see an example in Figure 2) and therefore the graph represents $S^{3}$.

(d) If $l=t=1$ then $\iota_{0}=\iota_{1}$. So $S_{m}(b, 1,1, \sigma, \tau)$ is the suspension of a surface.

As a consequence of point (b) and of the assumption $\operatorname{gcd}(l, t)=1$, we shall always suppose, without loss of generality, $1 \leq t \leq l$ and $t$ odd.

The necessary and sufficient condition for $G_{m}(b, l, t, \sigma, \tau)$ to represent a manifold will be investigated now. Let $\varphi$ and $\bar{\varphi}$ be the permutations ${ }^{2}$

$$
\begin{aligned}
& \varphi=\sigma^{\eta(t)} \tau^{\eta(2 t)} \sigma^{\eta(3 t)} \sigma^{\eta(4 t)} \cdots \sigma^{\eta((2 l-1) t)} \tau^{\eta(2 l t)}, \\
& \bar{\varphi}=\tau^{\eta(t)} \sigma^{\eta(2 t)} \tau^{\eta(3 t)} \sigma^{\eta(4 t)} \cdots \tau^{\eta((2 l-1) t)} \sigma^{\eta(2 l t)} .
\end{aligned}
$$

These permutations are very important in our contest because $\left(\iota_{3} \circ\right.$ $\left.\iota_{2}\right)^{l}(i, t)=(\varphi(i), t)$ and $\left(\iota_{2} \circ \iota_{3}\right)^{l}(i, 1)=(\bar{\varphi}(i), 1)$.

Let $H$ be a subgroup of $\Sigma_{b}$, then $H$ acts on $\mathbb{Z}_{b}$ dividing it into orbits. We denote by $c(H)$ the number of orbits of this action. If $S$ is a subset of $\Sigma_{b}$, we denote by $\langle S\rangle$ the subgroup of $\Sigma_{b}$ generated by $S$. In particular, if $\epsilon, \delta \in \Sigma_{b}$, we write $c(\epsilon)$ for $c(\langle\{\epsilon\}\rangle)$ and $c(\epsilon, \delta)$ for $c(\langle\{\epsilon, \delta\}\rangle)$. Furthermore, we use the notations $\delta^{\varepsilon}=\epsilon \delta \epsilon^{-1}$ and $[\delta, \epsilon]=\delta \epsilon \delta^{-1} \epsilon^{-1}$.

\footnotetext{
${ }^{2}$ Following our convention the product of permutations $\delta \epsilon$ means the composition of maps $\epsilon \circ \delta$.
} 


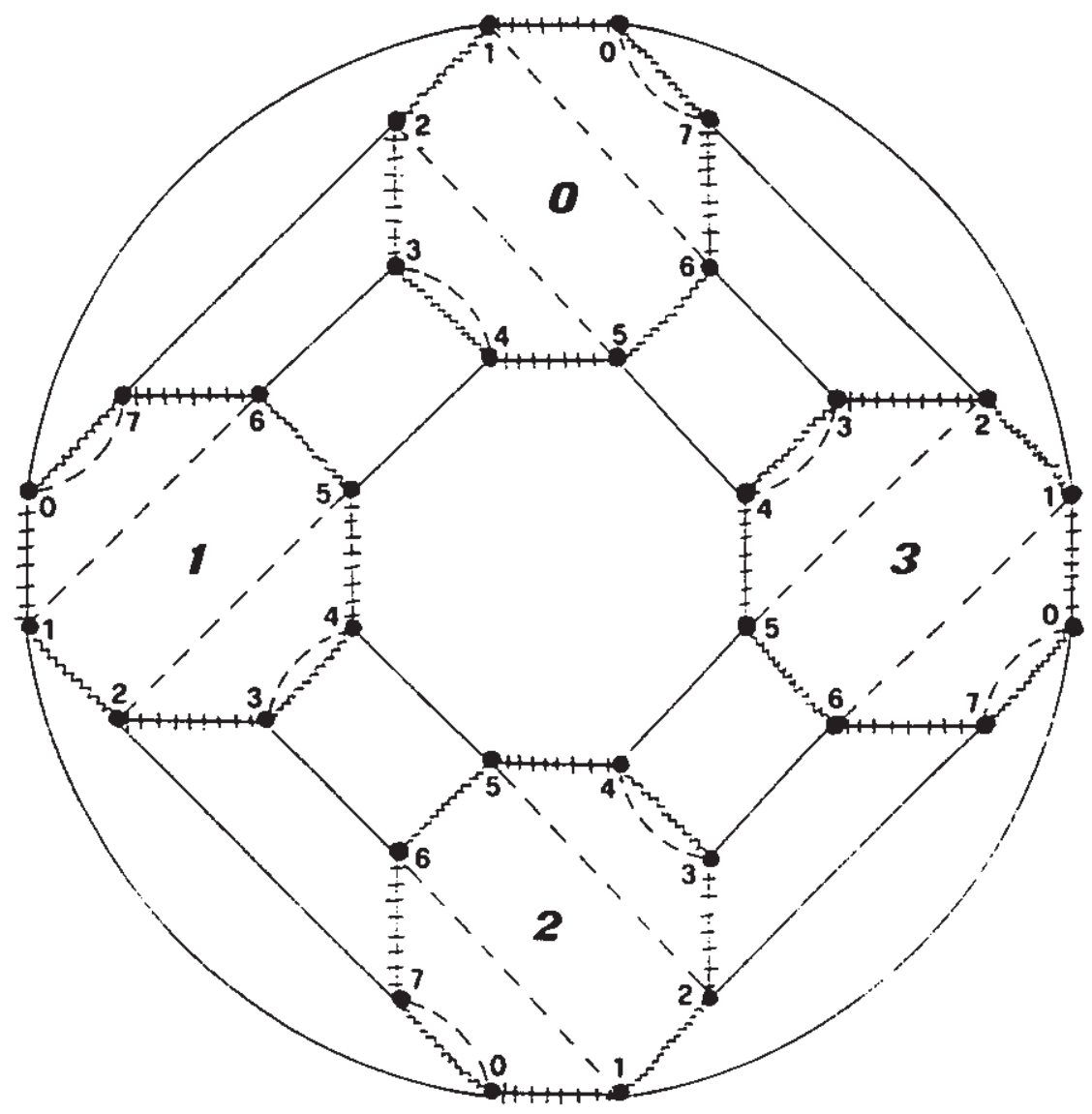

Figure 2. Planar regular embedding of $G_{m}\left(4,4,3,\left(\begin{array}{llll}0 & 1 & 2 & 3\end{array}\right), 1\right)-\xi=$ $\left(\begin{array}{llll}0 & 3 & 1 & 2\end{array}\right)-$

Note that $\bar{\varphi}$ is a conjugate of $\varphi^{-1}$, and precisely $\varphi^{-1}=\bar{\varphi}^{\mu}$, where $\mu=\tau^{\eta(t)} \sigma^{\eta(2 t)} \tau^{\eta(3 t)} \sigma^{\eta(4 t)} \cdots \sigma^{\eta\left(\left(t^{-1}-2\right) t\right)} \tau^{\left(\left(t^{-1}-1\right) t\right)}$. Thus, $\varphi=1$ iff $\bar{\varphi}=$ 1 ; moreover $c(\varphi)=c(\bar{\varphi})$. iff

Proposition 2.3. The graph $G_{m}(b, l, t, \sigma, \tau)$ represents a manifold

$$
c(\sigma)+c(\tau)+c(\varphi)=b+c(\sigma, \bar{\varphi})+c(\tau, \varphi)
$$


Proof. The statement is obtained by computing the number of 2 - and 3-residues of the graph. The calculation is similar to the one contained in [20] and we only give a brief sketch of it. For $i, j, k \in N_{3}$, let us denote by $r_{k}$ the number of $\left(N_{3}-\{k\}\right)$-residues and by $d_{i j}$ the number of $\{i, j\}$-residues of the graph. We get:

$$
\begin{gathered}
r_{3}=c(\sigma), r_{2}=c(\tau), r_{1}=c(\tau, \varphi), r_{0}=c(\sigma, \bar{\varphi}), \\
d_{01}=b, \quad d_{23}=c(\varphi), \\
d_{12}=\left\{\begin{array}{ll}
b \frac{l-2}{2}+2 c(\sigma) & \text { if } l \text { is even } \\
b \frac{l-1}{2}+c(\sigma) & \text { if } l \text { is odd }
\end{array}, d_{02}=\left\{\begin{array}{ll}
b \frac{l}{2} & \text { if } l \text { is even } \\
b \frac{l-1}{2}+c(\sigma) & \text { if } l \text { is odd }
\end{array},\right.\right. \\
d_{03}=\left\{\begin{array}{ll}
b \frac{l-2}{2}+2 c(\tau) & \text { if } l \text { is even } \\
b \frac{l-1}{2}+c(\tau) & \text { if } l \text { is odd }
\end{array}, d_{13}= \begin{cases}b \frac{l}{2} \\
b \frac{l-1}{2}+c(\tau) & \text { if } l \text { is odd }\end{cases} \right.
\end{gathered}
$$

So the number of 0-, 2- and 3-residues is $\alpha_{0}=2 b l, \alpha_{2}==b(2 l-$ $1)+2 c(\sigma)+2 c(\tau)+c(\varphi)$ and $\alpha_{3}=c(\sigma)+c(\tau)+c(\sigma, \bar{\varphi})+c(\tau, \varphi)$. Since $\alpha_{2}-\alpha_{0}-\alpha_{3}=c(\sigma)+c(\tau)+c(\varphi)-b-c(\sigma, \bar{\varphi})-c(\tau, \varphi)$, the statement follows.

Corollary 2.4. (a) If $\varphi=1$, then $G_{m}(b, l, t, \sigma, \tau)$ represents a manifold.

(b) If $\sigma$ and $\tau$ are cycles of onder $b$, then $G_{m}(b, l, t, \sigma, \tau)$ is a manifold iff $\varphi=1$.

(c) $G_{m}(b, 2,1, \sigma, \tau)$ represents a manifold iff

$$
c(\sigma)+c(\tau)+c([\sigma, \tau])=b+c\left(\sigma, \sigma^{\tau}\right)+c\left(\tau, \tau^{\sigma}\right) .
$$


Proof. (a) We have $c(\varphi)=b, c(\tau, \varphi)=c(\tau)$ and, since $\varphi=1$ iff $\bar{\varphi}=1, c(\sigma, \bar{\varphi})=c(\sigma)$.

(b) We have $c(\sigma)=c(\tau)=c(\sigma, \bar{\varphi})=c(\tau, \varphi)=1$. Since $c(\varphi)=b$ iff $\varphi=1$, we get the result.

(c) If $l=2$ and $t=1$, then $\varphi=[\sigma, \tau]$ and $\bar{\varphi}=[\tau, \sigma]$. Therefore $\langle\{\sigma, \bar{\varphi}\}\rangle=\left\langle\left\{\sigma, \sigma^{\tau}\right\}\right\rangle$ and $\langle\{\tau, \varphi\}\rangle=\left\langle\left\{\tau, \tau^{\sigma}\right\}\right\rangle$.

\section{TOPOLOGICAL PROPERTIES OF THE FAMILY}

The graph $G_{m}(b, l, t, \sigma, \tau)$ is highly symmetric and therefore, following rules (1) and (2) of Section 1 , we can construct the space $S_{m}(b, l, t, \sigma$, $\tau$ ) in a simple way. By performing on the $2 b l 3$-simplices of the space the identifications depending on $\iota_{0}$ and $\iota_{1}$, we obtain $b$ disjoint 3-balls $T_{0}, T_{1}, \ldots, T_{b-1}$. Let $\partial\left(T_{i}\right)$ be subdivided in four quarters of a sphere $R_{i}^{\prime}, R_{i}^{\prime \prime}, S_{i}^{\prime}$ and $S_{i}^{\prime \prime}$ as in Figure 3. Now, by the action of the 2edges, we have to identify the region $R_{i}^{t}$ with the region $R_{\sigma(i)}^{\prime \prime}$, for every $i \in \mathbb{Z}_{b}$, so that the points $A_{i}, B_{i}, C_{i}$ match respectively with the points $A_{\sigma(i)}, B_{\sigma(i)}, C_{\sigma(i)}$ and the point $P_{i, k}$ matches with the point $Q_{\sigma(i), k}$, for each $1 \leq k \leq l-1$. After these identifications, the space becomes a collection of $c(\sigma) 3$-balls. The last operation, depending on $\iota_{3}$, is to identify the region $S_{i}^{\prime \prime}$ with the region $S_{\tau(i)}^{\prime}$, for every $i \in \mathbb{Z}_{b}$, so that the points $P_{i-1, l-t}, D_{i}, P_{i, t}$ match respectively with the points $P_{\tau(i)-1, l-t}, D_{\tau(i)}$, $P_{\tau(i), t}$ and the arc $P_{i, t} C P_{i-1, l-t}$ matches with the arc $P_{\tau(i), t} B P_{\tau(i)-1, l-t}$. 


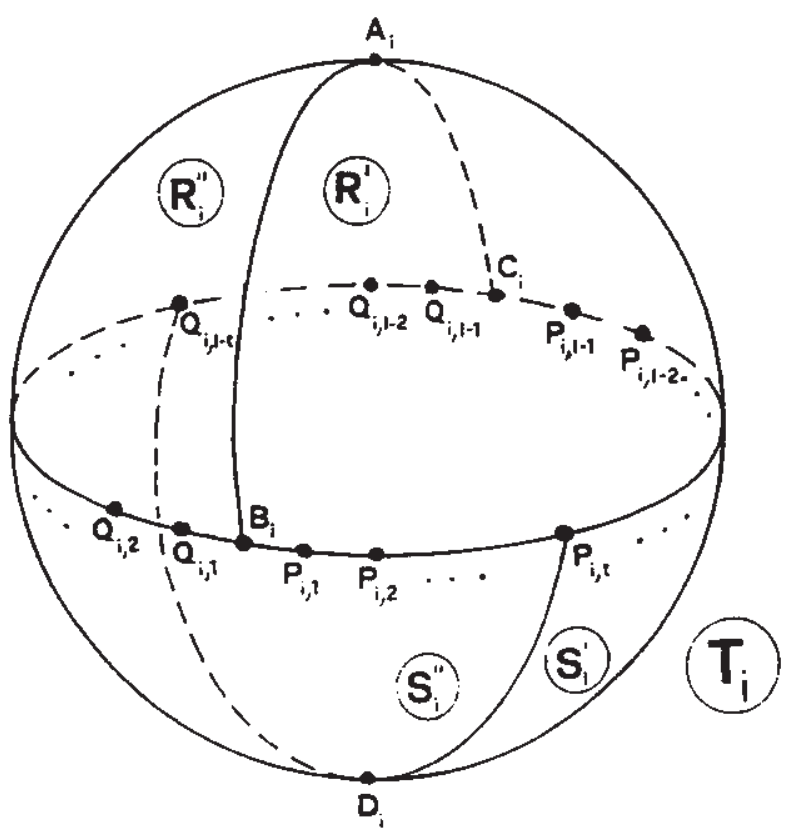

Figure 3.

We shall study some topological properties of the spaces of $\mathcal{S}_{m}$ as branched coverings of $S^{3}$.

The map $f: \mathbb{Z}_{b} \times \mathbb{Z}_{2 l}=V\left(G_{m}(b, l, t, \sigma, \tau)\right) \rightarrow\{0\} \times \mathbb{Z}_{2 l}=$ $V\left(G_{m}(1, l, t, 1,1)\right)$ defined by

$$
f(i, j)=(0, j)
$$

is a cp-morphism and, if $b>1$, a 1-covering. Therefore

$$
|f|: S_{m}(b, l, t, \sigma, \tau) \rightarrow S_{m}(1, l, t, 1,1) \cong S^{3}
$$

is a $b$-fold branched covering map. From now on we always assume $b>1$. In order to completely describe $|f|$ we shall obtain the branching set $B_{|f|}$ (Proposition 3.2) and the monodromy map $\omega_{|f|}$ (Proposition 3.4).

Since $\operatorname{gcd}(l, t)=1$, the graph $G_{m}(1, l, t, 1,1)$ has only one $\{2,3\}$ residue and we denote this residue by $\Theta$. Figure 4 shows two examples. 

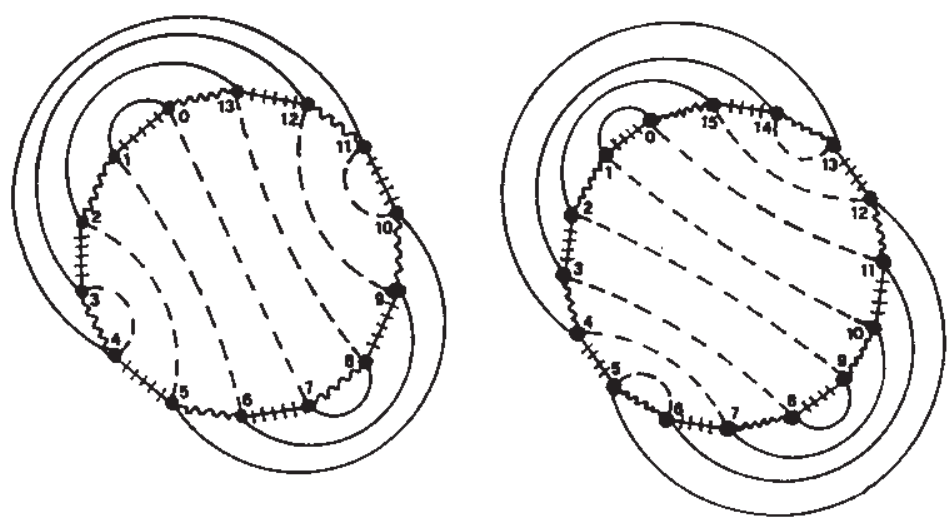

Figure 4. $G_{m}(1,7,3,1,1)$ and $G_{m}(1,8,5,1,1)$

Lemma 3.1. The 2-residues of $G=G_{m}(1, l, t, 1,1)$ not ordinarily covered by $f$ are the following:

(i) if $\sigma \neq 1$ : the $\{1,2\}$-residue containing $(0,0)$ and the $\{0,2\}$-residue (resp. the $\{1,2\}$-residue) containing $(0, l)$ when $l$ is odd (resp. when $l$ is even), both of length two;

(ii) if $\tau \neq 1$ : the $\{0,3\}$-residue containing $(0, t+1)$ and the $\{1,3\}$ residue (resp. the $\{0,3\}$-residue) containing $(0, l+t+1)$ when $l$ is odd (resp. when l is even), both of length two;

(iii) if $\varphi \neq 1$ : the $\{2,3\}$-residue $\Theta$.

Proof. (i) If $\sigma \neq 1$ then there exists $i \in \mathbb{Z}_{b}$ such that $\sigma(i) \neq i$. Since $\iota_{2} \circ \iota_{1}(i, 0)=(\sigma(i), 0)$ and, when $l$ is odd (resp. when $l$ is even) $\iota_{2} \circ \iota_{0}(i, l)=\left(\sigma^{-1}(i), l\right)$ (resp. $\left.\iota_{2} \circ \iota_{1}(i, l)=\left(\sigma^{-1}(i), l\right)\right)$, the 2-residues of $G$ containing $(i, 0)$ and $(i, 1)$ respectively, and covering the above residues, have length greater than two.

(ii) Let $i \in \mathbb{Z}_{b}$ such that $\tau(i) \neq i$. Since $\iota_{3} \circ \iota_{0}(i, t+1)=(\tau(i), t+1)$ and, when $l$ is odd (resp. when $l$ is even) $\iota_{3} \circ \iota_{1}(i, l+t+1)=\left(\tau^{-1}(i), l+\right.$ $t+1)\left(\right.$ resp. $\left.\iota_{3} \circ \iota_{0}(i, l+t+1)=\left(\tau^{-1}(i), l+t+1\right)\right)$, we obtain the same result of point (i).

(iii) $\Theta$ has length $2 l$. Since $\left(\iota_{3} \circ \iota_{2}\right)^{l}(i, t)=(\varphi(i), t)$ it is covered by at least one $\{2,3\}$-residue of $G_{m}(b, l, t, \sigma, \tau)$ of length greater than $2 l$.

To complete the proof we have to show that the remaining 2-residues are ordinarily covered by $f$. First we note that the unique $\{0,1\}$-residue of $G$ has length $2 l$ and is covered by $b$ residues of length $2 l$. Finally, the remaining residues are of length four and are covered by residues all of length four. 
Let $L$ be the 1-subcomplex associated by the bijection $\Psi$ (see Section 1) to the five 2-residues listed in the previous lemma. Incidence arguments on the lattice of the residues of $G_{m}(1, l, t, 1,1)$ show that $|L|$ is homeomorphic to a $\theta-g r a p h$ when $l$ is odd and is homeomorphic to a handcuff-graph when $l$ is even (see Figure 5). Of course, we are interested in determining how $|L|$ is embedded in $S^{3}$. Observe that the embedding only depends on $l$ and $t$; we denote it by $g(l, t)$. Figure 6 shows how this graph is embedded in the 3 -sphere and a constructive proof of the result is contained in the Appendix. Note that, by removing from $g(l, t)$ the 1 -simplex $\tilde{s}$ associated to $\Theta$, we get a two-bridge knot or $\operatorname{link}^{3} b(l, t)$. Furthermore, $g(l, t)$ has a trivial embedding iff $l=1=t$.
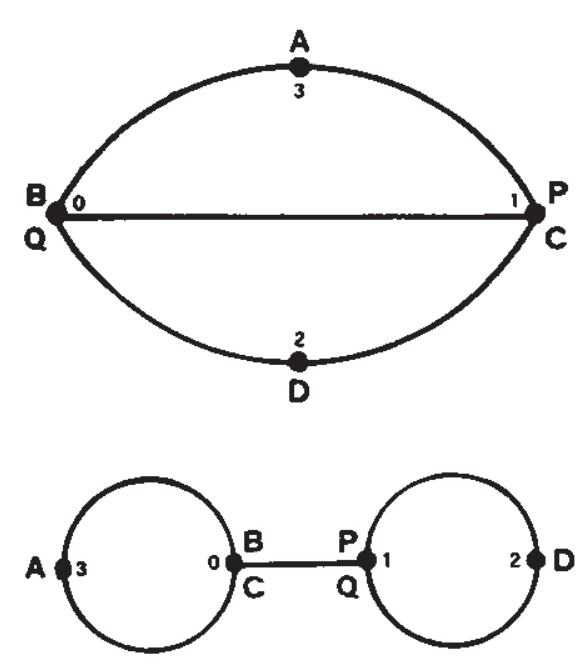

Figure 5. A $\theta$-graph and a handcuff-graph (trivially embedded)

Remark 3.1. The coefficients $a_{i}$ of Figure 6, called Conway symbols, are uniquely obtained by the following algorithm:

$$
l=a_{1} r_{0}+r_{1}
$$

3 For the conventions on two-bridge knots and links we refer to [2] and denote by $b(l, t)$ the two-bridge knot or link of type $(l, t)$. 


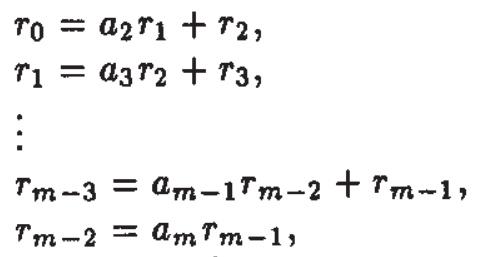

where $r_{0}=t$ and $0 \leq r_{i}<r_{i-1}$ when $i \geq 1$.

Now we are able to describe the branching set $B_{|f|}$.

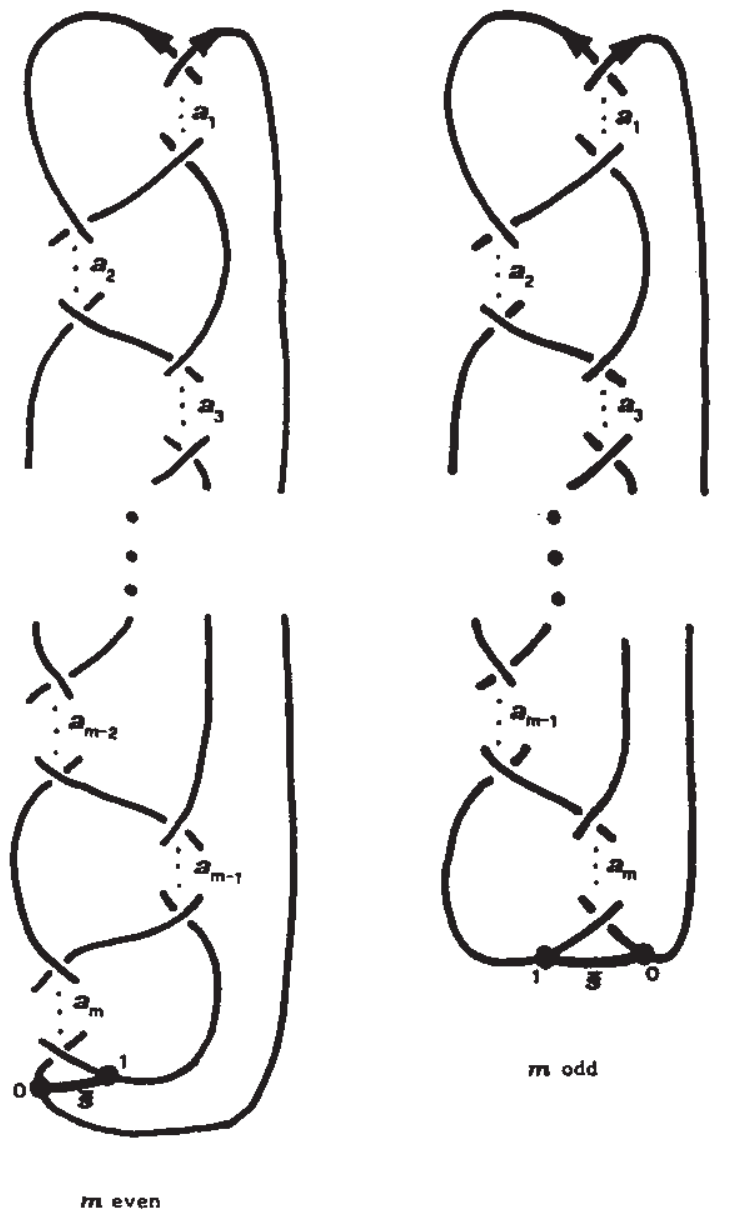

Figure 6. The graph $g(l, t)$ - twists of $\pi$ radiants - 
Proposition 3.2. Let $B=B_{|f|}$ be the branching set of $|f|$.

1) Let us suppose l odd:

a) if $\sigma, \tau, \varphi \neq 1$, then $B$ is the $\theta$-graph $g(l, t)$ of Figure 6 ;

b) if $\varphi=1$, then $B$ is the two-bridge knot $b(l, t)$;

c) if $\sigma=1$ or $\tau=1$, then $B$ is the trivial knot.

2) Let us suppose l even:

a) if $\sigma, \tau, \varphi \neq 1$, then $B$ is the handcuff-graph $g(l, t)$ of Figure 6 ;

b) if $\varphi=1$, then $B$ is the two-bridge link $b(l, t)$;

c) if $\sigma=1$ or $\tau=1$, then $B$ is the trivial knot.

Proof. If $\sigma, \tau, \varphi \neq 1$, then $B$ is the subcomplex associated to the five 2-residues of Lemma 3.1. Therefore, $B=g(l, t)$. If $\varphi=1$, then $B$ does not contain the 1 -simplex associated to $\Theta$. Hence, $B$ is just $b(l, t)$. Finally, let us suppose $\sigma=1$ or $\tau=1$. In this case, $|f|$ is a cyclic covering and, since $S_{m}(b, l, t, \sigma, \tau) \cong S^{3}$ by Lemma 2.2.c, the branching set $B$ must be a trivial knot.

Remark 3.2. Theorem 3.2 gives a topological proof to Corollary 2.4.a. In fact, if $\varphi=1$ the space $S_{m}(b, l, t, \sigma, \tau)$ is a branched covering of a knot or of a link and therefore is a manifold. The condition $\varphi=1$ is only sufficient because also a branched covering of the graph $g(l, t)$ can be a manifold. For example, if $b=4, l=3, t=1, \sigma=\left(\begin{array}{ll}0 & 1\end{array}\right)\left(\begin{array}{ll}2 & 3\end{array}\right)$ and $\tau=\left(\begin{array}{ll}0 & 2\end{array}\right)\left(\begin{array}{ll}13\end{array}\right)$, then $\varphi=\left(\begin{array}{ll}03\end{array}\right)\left(\begin{array}{ll}1 & 2\end{array}\right)=\bar{\varphi}$ and therefore the branching set is $g(3,1)$. But $S_{m}(4,3,1, \sigma, \tau)$ is a manifold, since $c(\sigma)=c(\tau)=c(\varphi)=2$ and $c(\tau, \varphi)=c(\sigma, \bar{\varphi})=1$.

To determine the fundamental group of $S^{3}-g(l, t)$ we need to choose a suitable splitting complex for $g(l, t)$. It is easy to see that the 2subcomplex $\tilde{K}$ consisting of the 2-simplices associated to the 2- and 3 edges of $G_{m}(1, l, t, 1,1)$ is a good choice. In fact $g(l, t)$ is contained in $\tilde{K}$ because it consists of 1 -simplices associated by $\Psi$ to 2 -residues containing 2 - or 3 -edges. Moreover, $\tilde{K}$ is simply-connected because it is the internal of the 3-ball $T_{0}$ previously described. From Theorem 3.3.1 of [22] follows that $\pi_{1}\left(S^{3}-g(l, t)\right)$ admits a presentation with generators associated to the set of 3-edges $\left\{x_{j}=(\{(0, j+t),(0,1-j+t)\}, 3) \mid 1 \leq j \leq l\right\}$ and the set of 2-edges $\left\{y_{j}=(\{(0, j),(0,1-j)\}, 2) \mid 1 \leq j \leq l\right\}$, while the relators are in 1-1 correspondence with the $\{0,2\}-\{1,2\}-\{0,3\}$ - and $\{1,3\}$ residues not representing $g(l, t)$. We orient each $x_{j}$ from $(0,1-j+t)$ 
to $(0, j+t)$ and each $y_{j}$ from $(0, j)$ to $(0,1-j)$. The $l-1\{1,3\}$ - and $\{0,3\}$-residues (all of length four) give the relations $x_{1}=x_{2}=\cdots=x_{l}$ $(=X)$. In the same way, from the $l-1\{1,2\}$ - and $\{0,2\}$-residues (again of lenght four), we get $y_{1}=y_{2}=\cdots y_{i}(=Y)$. Since there are no other relations the following result is proved:

Proposition 3.3. The fundamental group of $S^{3}-g(l, t)$ is the free group on two generators

$$
\pi_{1}\left(S^{3}-g(l, t)\right)=\langle\{X, Y\} ; \emptyset\rangle,
$$

for all $l$ and $t$.

When the branching set $B_{|f|}$ is different from $g(l, t)$, we can describe the fundamental group of $S^{3}-B_{|f|}$ again with the two generators $X$ and $Y$, but also with some relators corresponding to the 1-simplices of $g(l, t)$ not contained in $B_{|f|}$. In particular, when $B_{|f|}=b(l, t)$, we get a relator $r$ corresponding to $\Theta$. By walking along this residue, starting by the 2-edge from $(0, t)$, we obtain

$$
r=Y^{\eta(t)} X^{\eta(2 t)} Y^{\eta(3 t)} X^{\eta(4 t)} \ldots Y^{\eta((2 l-1) t)} X^{\eta(2 l t)} .
$$

On the contrary, when $B_{|f|}$ is the trivial knot we obtain the relation $X=1$ (or $Y=1$ ), and the fundamental group is isomorphic to $\mathbb{Z}$. The monodromy map $\omega_{|f|}$ can now be obtained.

Proposition 3.4. The monodromy map $\omega_{|f|}: \pi_{1}\left(S^{3}-B_{|f|}\right)=$ $\langle\{X, Y\} ; R\rangle \rightarrow \Sigma_{b}$, associated to the covering $|f|$, is defined by:

$$
\begin{aligned}
& \omega_{|f|}(X)=\tau, \\
& \omega_{|f|}(Y)=\sigma .
\end{aligned}
$$

Proof. First we observe that $\omega_{|f|}$ is well-defined also when $B_{|f|} \neq$ $g(l, t)$ and therefore $R \neq \emptyset$. In fact if $B_{|f|}=b(l, t)$ then $R=\{r\}$ and $\omega_{\bar{f}}(r)=\varphi=1$. Moreover, if $B_{|f|}$ is the trivial knot then we obtain the same conclusion because $X=1$ iff $\tau=1$ and $Y=1$ iff $\sigma=1$. Let us fix as base point $O$ of $\pi_{1}\left(S^{3}-B_{|f|}\right)$ the vertex $(0,0)$ of the 3 -gem $G=G_{m}(1, l, t, 1,1)$, embedded in $S^{3}$ as 1-skeleton of the ball-complex dual to $K(G)$. The loops $X, Y \in \pi_{1}\left(S^{3}-B_{|f|}, O\right)$ are showed in Figure 7 . Let $|f|^{-1}(O)=\left\{O_{i} \mid i \in \mathbb{Z}_{b}\right\}$ be the fiber of $O$, where $O_{i}$ is the barycentre 
of the 3-simplex associated to the vertex $(i, 0)$ of $G_{m}(b, l, t, \sigma, \tau)$. Then the lifting of $X$ (resp. of $Y$ ) starting at $O_{i}$ is an arc ending in $O_{\tau(i)}^{\prime}$ (resp. in $O_{\sigma(i)}^{\prime}$ ). This completes the proof.

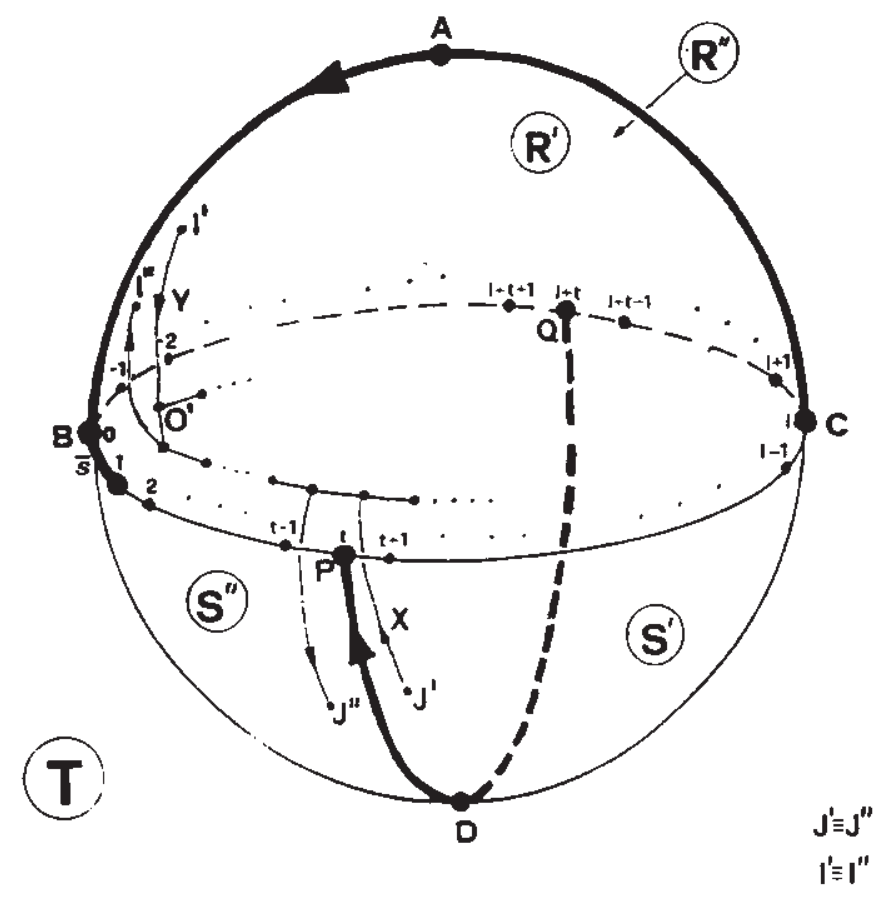

Figure 7. $\left(I^{\prime} \in R^{\prime \prime}, I^{\prime \prime} \in R^{\prime}, J^{\prime} \in S^{\prime}, J^{\prime \prime} \in S^{\prime \prime}\right)$

As consequences of the above results we have the following characterization of the spaces of $\mathcal{S}_{m}$ :

Theorem 3.5. The class of spaces $\mathcal{S}_{m}$ is the class of all the coverings of $S^{3}$, branched over either $g(l, t)$ or $b(l, t)$.

Corollary 3.6. Let $M$ be a manifold which is a branched covering of a trivially embedded $\theta$-graph, then $M \simeq S^{3}$. 
Proof. A branched covering of $g(1,1)$ is homeomorphic to $S_{m}(b, 1$, $1, \sigma, \tau)$, for suitable $b, \sigma, \tau$. Therefore the result follows from Lemma 2.2.d.

Corollary 3.7. Let $l$ be even, then the following properties hold:

(i) thene exist no abelian coverings of $g(l, t)$;

(ii) if $\sigma \tau=\tau \sigma$ then the space $S_{m}(b, l, t, \sigma, \tau)$ is a 3-manifold.

Proof. (i) Let $e(j)=\sum_{k=0}^{i-1} \eta(j+2 k t)$. It is simple to check that $e(j)=0$ for each $j \in \mathbb{Z}_{2 l}$. Now, let $Z$ be any meridian around the 1simplex $\bar{s}$ of $g(l, t)$ associated to the 2-residue $\Theta$. Then, $Z$ is mapped by the monodromy to (a conjugate of) $\varphi$ or $\varphi^{-1}$. Since $\sigma \tau=\tau \sigma$, we obtain $\varphi=\sigma^{e(t)} \tau^{e(2 t)}=1$. Therefore, $\omega(Z)=1$ and the simplex $\bar{s}$ cannot be contained in the branching set.

(ii) By (i), $S_{m}(b, l, t, \sigma, \tau)$ is the branched covering either of $b(l, t)$ or of the trivial knot. Hence, the space is a manifold.

In Proposition 7 of [4], A. Cavicchioli states that if the condition

$$
\begin{gathered}
\alpha^{-\eta(j+(2 l-1) t)}\left(\ldots\left(\alpha^{-\eta(j+3 t)}\left(\alpha^{-\eta(j+t)}(i+\eta(j))+\eta(j+2 t)\right) \ldots\right)\right. \\
+\eta(j+(2 l-2) t))=i
\end{gathered}
$$

holds for every $j \in \mathbb{Z}_{2 l}$, then $G_{a}(b, l, t, \alpha)=G_{m}\left(b, l, t, v, \alpha^{-1}\right)$ is a crystallization and therefore represents a manifold. Now we give a topological interpretation of this condition. First we claim that if (2.5) holds for a value of $j$ then it holds for every $j \in \mathbb{Z}_{2 l}$. In fact (2.5), written in terms of permutations with $\tau=\alpha^{-1}$, becomes

$$
v^{\eta(j)} \tau^{\eta(j+t)} v^{\eta(j+2 t)} \tau^{\eta(j+3 t)} \cdots v^{\eta(j+(2 l-2) t)} \tau^{\eta(j+(2 l-1) t)}=1 .
$$

Replacing $j$ with $j+1$, taking the inverse and recalling that $-\eta(1+j)=$ $\eta(-j)=\eta(2 l-j)$, we obtain 


$$
\begin{gathered}
\tau^{\eta(-j+t)} v^{\eta(-j+2 t)} \tau^{\eta(-j+3 t)} v^{\eta(-j+4 t)} \ldots \\
\tau^{\eta(-j+(2 l-1) t)} v^{\eta(-j+2 l t)}=1 .
\end{gathered}
$$

Now set $k=t^{\prime} j$, where $t^{\prime}$ is the inverse of $t$ in $\mathbb{Z}_{l}$, then $-j+2 k t=j$ (in $\left.\mathbb{Z}_{2 l}\right)$ and therefore, conjugating by $\tau^{\eta(-j+t)} v^{\eta(-j+2 t)} \ldots \tau^{\eta(-j+(2 k-1) t)}$, we again obtain (2.6). Evaluating (2.6) for $j=t$, we get $\varphi=1$. Thus, (2.5) is equivalent to the condition $\varphi=1$ and therefore it is just a sufficient condition for the branching set to be a knot or a link. This guarantees that the represented space is a manifold. By making use of Corollary 2.4.b, we can prove that, in the Cavicchioli family, the condition is also necessary.

Proposition 3.8. A graph $G_{a}(b, l, t, \alpha) \in \mathcal{G}_{a}$ represents a manifold iff condition (2.5) holds. So every gem of $\mathcal{G}_{a}$ is also a crystallization.

Proof. By Lemma 2.1.c, $G_{a}(b, l, t, \alpha)$ is the graph $G_{m}(b, l, t, v$, $\alpha^{-1}$ ). Since $\alpha$ and $v$ are both cycles of order $b$, the statement follows from Corollary 2.4.b.

\section{A "UNIVERSAL" CLASS OF 4-COLOURED GRAPHS}

For each $k \geq 1$, let $\mathcal{G}_{m}^{k}$ denote the class of 4-coloured graphs $\mathcal{G}_{m}^{k}=$ $\left\{G_{m}(b, l, t, \sigma, \tau) \in \mathcal{G}_{m} \mid l=k\right\}$ and let $\mathcal{S}_{m}^{k}$ denote the class of spaces $\mathcal{S}_{m}^{k}=\left\{S_{m}(b, l, t, \sigma, \tau) \in \mathcal{S}_{m} \mid G_{m}(b, l, t, \sigma, \tau) \in \mathcal{G}_{m}^{k}\right\}$.

In this section we show that the class $\mathcal{G}_{m}^{2}$ is "universal": every 3 dimensional orientable manifold or quasi-manifold can be represented by a graph belonging to $\mathcal{G}_{m}^{2}$. In particular, we claim that $S_{m}(b, 2,1, \sigma, \tau)$ is homeomorphic to the (singular) manifold $N(\sigma, \tau)$ introduced by J.M. Montesinos in [18]. In that paper the Author proved that the graph $G$ of Figure 8 is universal; i.e. every orientable singular manifold is a branched covering of $G$. Since $\pi_{1}\left(S^{3}-G\right)$ is a free group on two generators, the monodromy map is completely defined by a transitive pair of permutations $\sigma, \tau \in \Sigma_{b}$. Referring to Figure 8 (it is just a copy 
of Figure 2 of [18]), the monodromy sends the generators $x$ and $y$ of $\pi_{1}\left(S^{3}-G\right)$ respectively to $\sigma$ and to $\tau$.

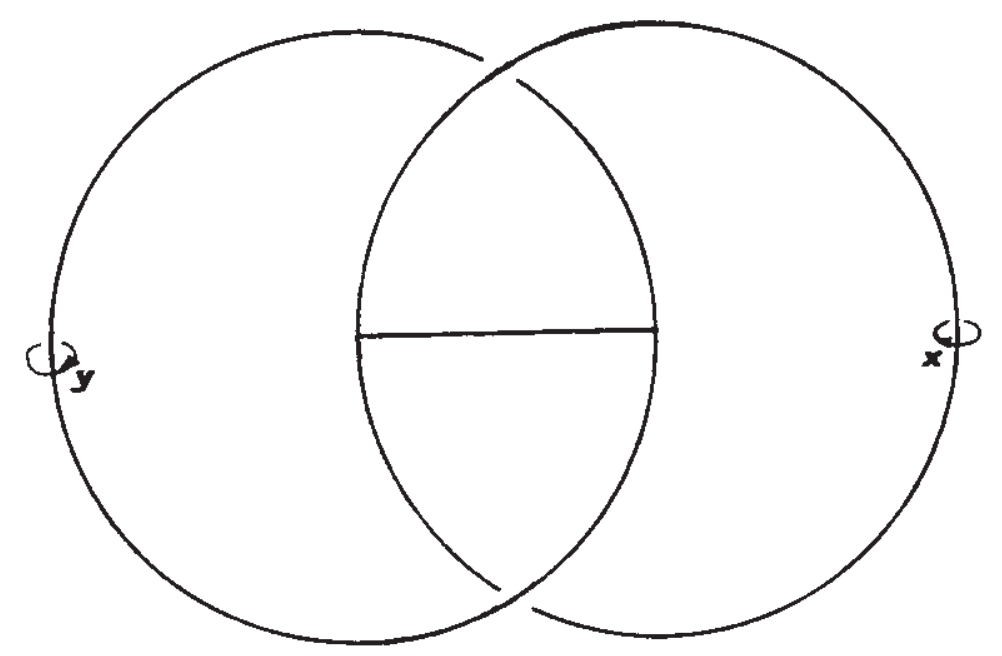

Figure 8. The Montesinos universal graph

Proposition 4.1. (a) The graph $g(2,1)$ is the Montesinos universal graph $G$.

(b) The singular manifold $S_{m}(b, 2,1, \sigma, \tau)$ is homeomorphic to $N(\sigma$, $\tau)$, for every transitive pair $(\sigma, \tau)$ of permulations of $\Sigma_{b}$.

Proof. Figure 9 shows the construction of $g(2,1)$ by a sequence of identifications. Moreover the loops $X$ and $Y$ are homotopic respectively to the loops $y$ and $x$ of Figure 8.

Remark 4.1. Note the correspondence between Corollary 7 of [18] and Corollary 2.4.c.

The above result allows us to obtain a "universal" class of 4-coloured graphs representing all orientable 3 -dimensional singular manifolds. 

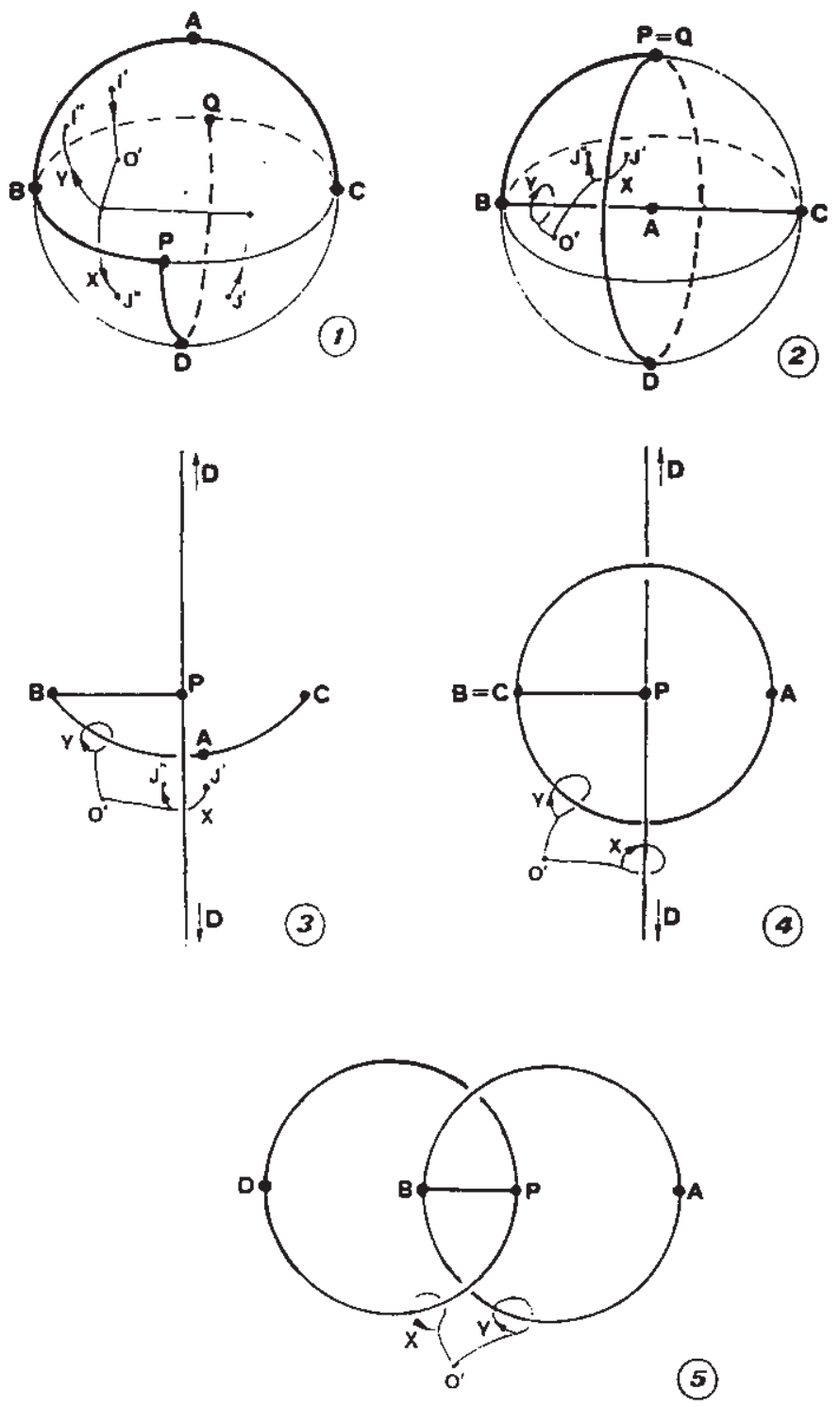

Figure 9. Construction of $g(2,1)$ 
Corollary 4.2. Every 3-dimensional orientable singular manifold $\tilde{M}$ belongs to $\mathcal{S}_{m}^{2}$. Hence there exists an integer $b>0$ and a transitive pair $(\sigma, \tau)$ of permutations of $\Sigma_{b}$ such that $G_{m}(b, 2,1, \sigma, \tau)$ represents $\tilde{M}$.

Figure 10 shows an example for the manifold $S^{1} \times S^{2}$.

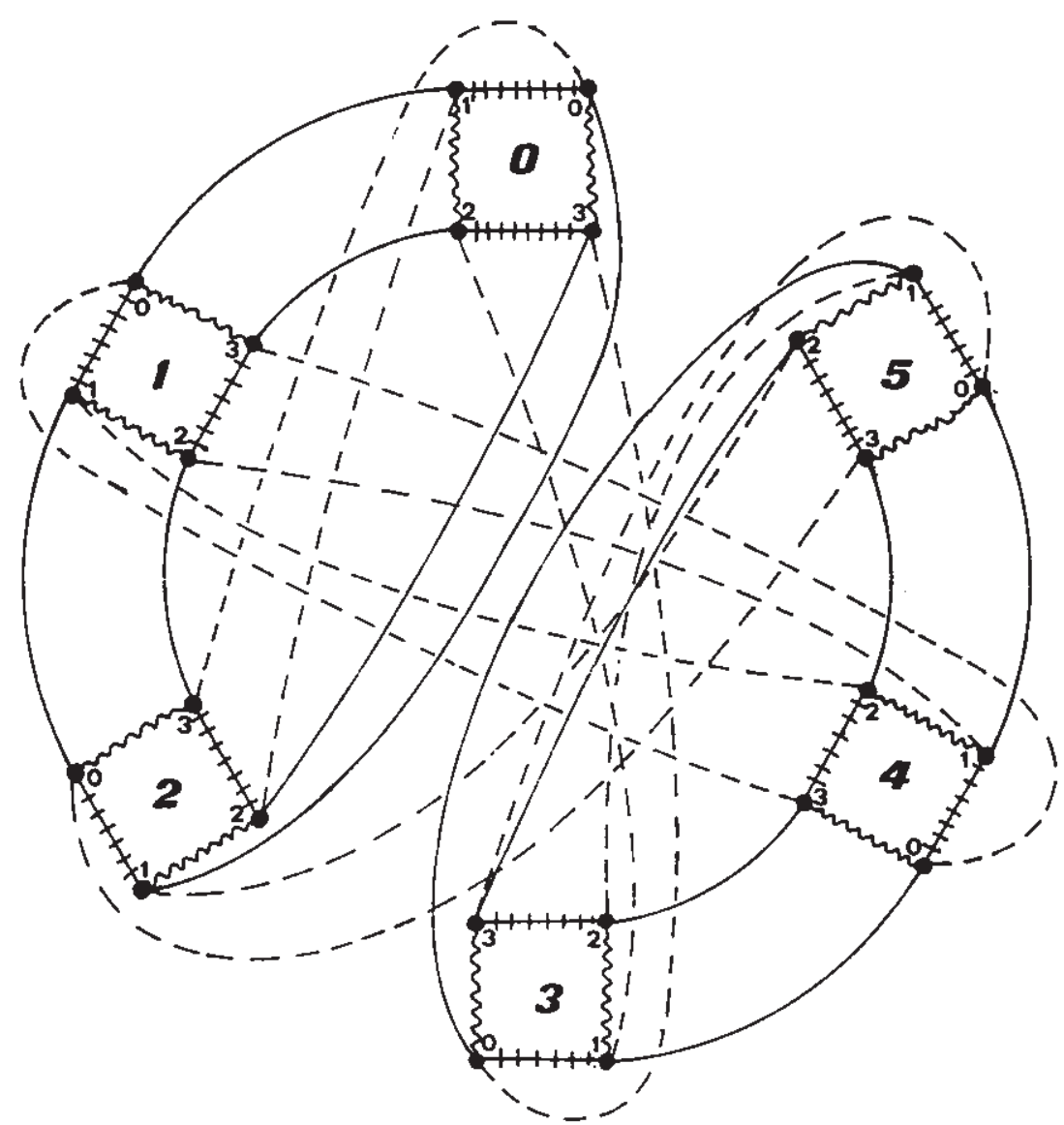

Figure 10. $G_{m}\left(6,2,1,\left(\begin{array}{lll}0 & 1 & 2\end{array}\right)\left(\begin{array}{lll}3 & 4 & 5\end{array}\right),\left(\begin{array}{llll}0 & 2 & 5 & 3\end{array}\right)\left(\begin{array}{ll}1 & 4\end{array}\right)\right)$-represents $S^{2} \times S^{1}$ - 
Remark 4.2. An algorithm for obtaining, from a bipartite crystallization $\Omega$, a transitive permutation pair $(\sigma, \tau)$ such that $|K(\Omega)| \cong$ $N(\sigma, \tau)$ is contained in [5] and [26]. The algorithm has been extended to the general case of bipartite 4-coloured graphs by L. Grasselli in [12].

\section{A. APPENDIX}

The embedded graph $g(l, t)$ of Figure 6, will be constructed from $G=G_{m}(1, l, t, 1,1)$ by using just rules (1) and (2) of Section 1.

By performing on the 3-simplices of $K(G)$ only the identifications depending on the 0 - and 1-edges of $G$, the 3-ball $T$ of Figure 7 is obtained. We refer to the Conway symbols and the coefficients $m, r_{1}, r_{2}, \ldots, r_{m-1}$ described in Remark 3.1.

By the identifications depending on the 3-edges, we glue the two quarters $S^{\prime}$ and $S^{\prime \prime}$ of the 2-sphere $\partial(T)$ together and we obtain a new 3-ball $T^{\prime}$. If the point $A$ goes to infinity, the ball becomes homeomorphic to the half space $z \leq 0$ of $\mathbb{R}^{3}$. The boundary $\partial\left(T^{\prime}\right)$ becomes the plane $z=0$, with $A=\infty$. In Figure A.1, $T^{\prime}$ is depicted, with $\partial\left(T^{\prime}\right)$ represented by the plane of the paper. At this stage the simplices of $g(t, t)$ are marked by the thick line. Note that the open arc $P Q$ belongs to the open half space $z<0$, whereas the remainder of the graph belongs to the plane $z=0$. Now we perform on $\partial\left(T^{\prime}\right)$ the glueings depending on the 3-edges. The $t$ 3-edges incident to the vertices $(0, l-j$ ) (for $0 \leq j \leq t-1$ ) of the graph, match the region $\alpha_{1}^{\prime}$ with the region $\alpha_{1}^{\prime \prime}$. So we obtain a new 3-ball which is shown in Figure A.2. Observe that now the open $\operatorname{arc} C A$ belongs to the interior of the ball. By the action of the $t$ 2-edges adjacent to the vertices $(0, l-t-j$ ) (for $0 \leq j \leq t-1$ ) we match the regions $\alpha_{2}^{\prime}$ and $\alpha_{2}^{\prime \prime}$, and so on. After $a_{1}-1$ steps, we obtain either the 3 -ball of Figure A.3, if $t$ divides $l$ (and therefore $t=1=m$ ), or, otherwise, the ball of Figure A.4. In the former case the remaining 3-edge glues the two hemispheres $\alpha^{t}$ and $\alpha^{\prime \prime}$ of the boundary of the ball together, so obtaining a 3-sphere, but without changing the embedding of $g(l, t)$. In the latter case we have to do another stage of identifications. By the $r_{1}$ 2-edges adjacent to the vertices $(0, t-j)$ (for $0 \leq j \leq r_{1}-1$ ), we glue the regions $\beta_{1}^{\prime}$ and $\beta_{1}^{\prime \prime}$ together and we obtain the 3-ball of Figure A.5. By the $r_{1}$ 2-edges incident to the vertices $\left(0, t-r_{1}-j\right.$ ) (for $0 \leq j \leq r_{1}-1$ ), we glue the regions $\beta_{2}^{\prime}$ and $\beta_{2}^{\prime \prime}$ together and so on. At the end of this stage we arrive, after $a_{2}$ steps, either to the 3-ball of Figure A.6, when 
$r_{1}$ divides $t$ (and therefore $r_{1}=1$ and $m=2$ ), or, otherwise, to the one of Figure A.7. In the former case, $g(l, t)$ is obtained, otherwise in the latter case we need a new stage of identifications (see Figures A.8 and A.9) and so on. Of course the process will finish after $m$ stages and therefore the embedded graph $g(l, t)$ will be equivalent to one of the two cases of Figure 6, depending on the parity of $m$.
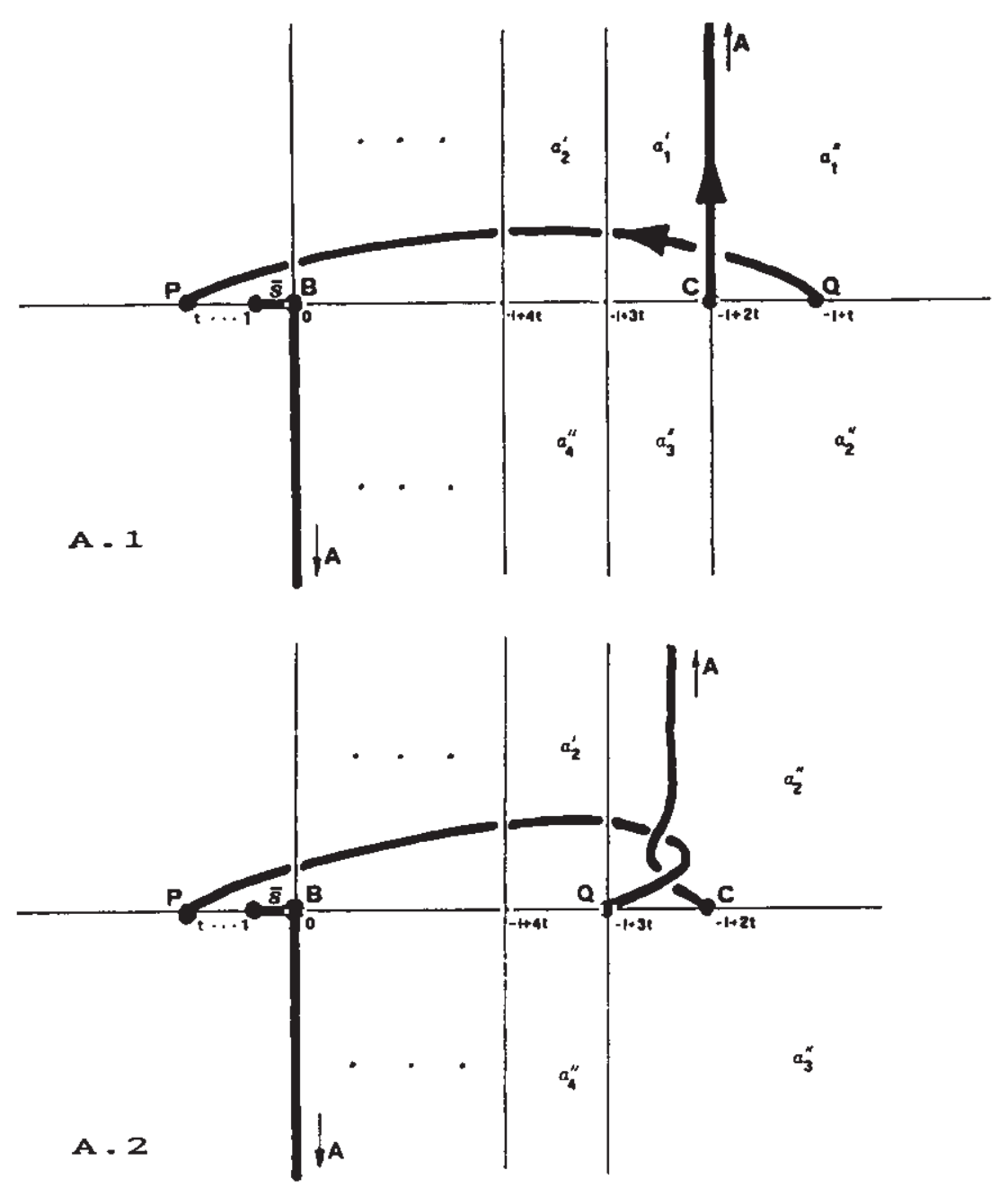


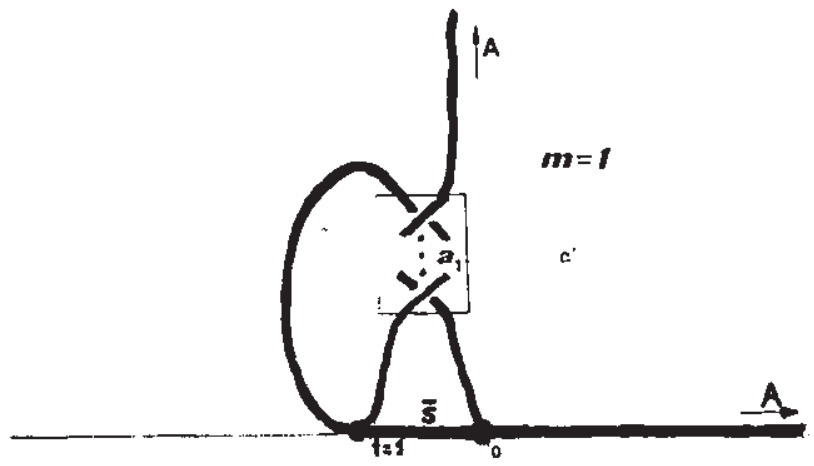

A. 3

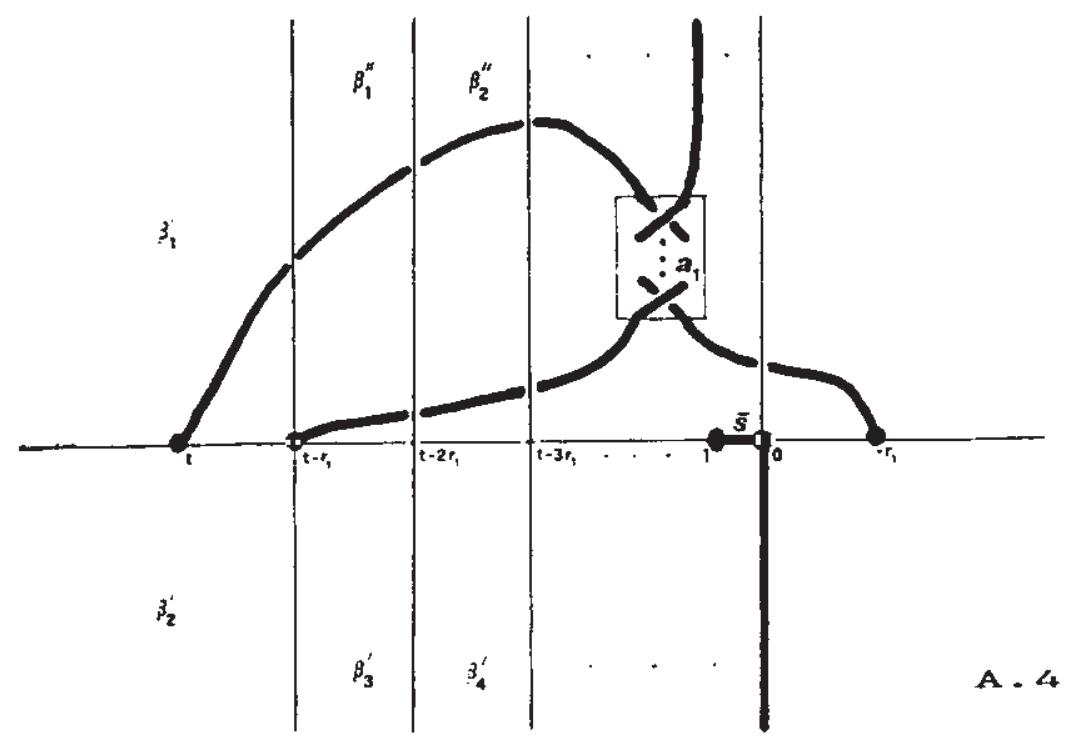



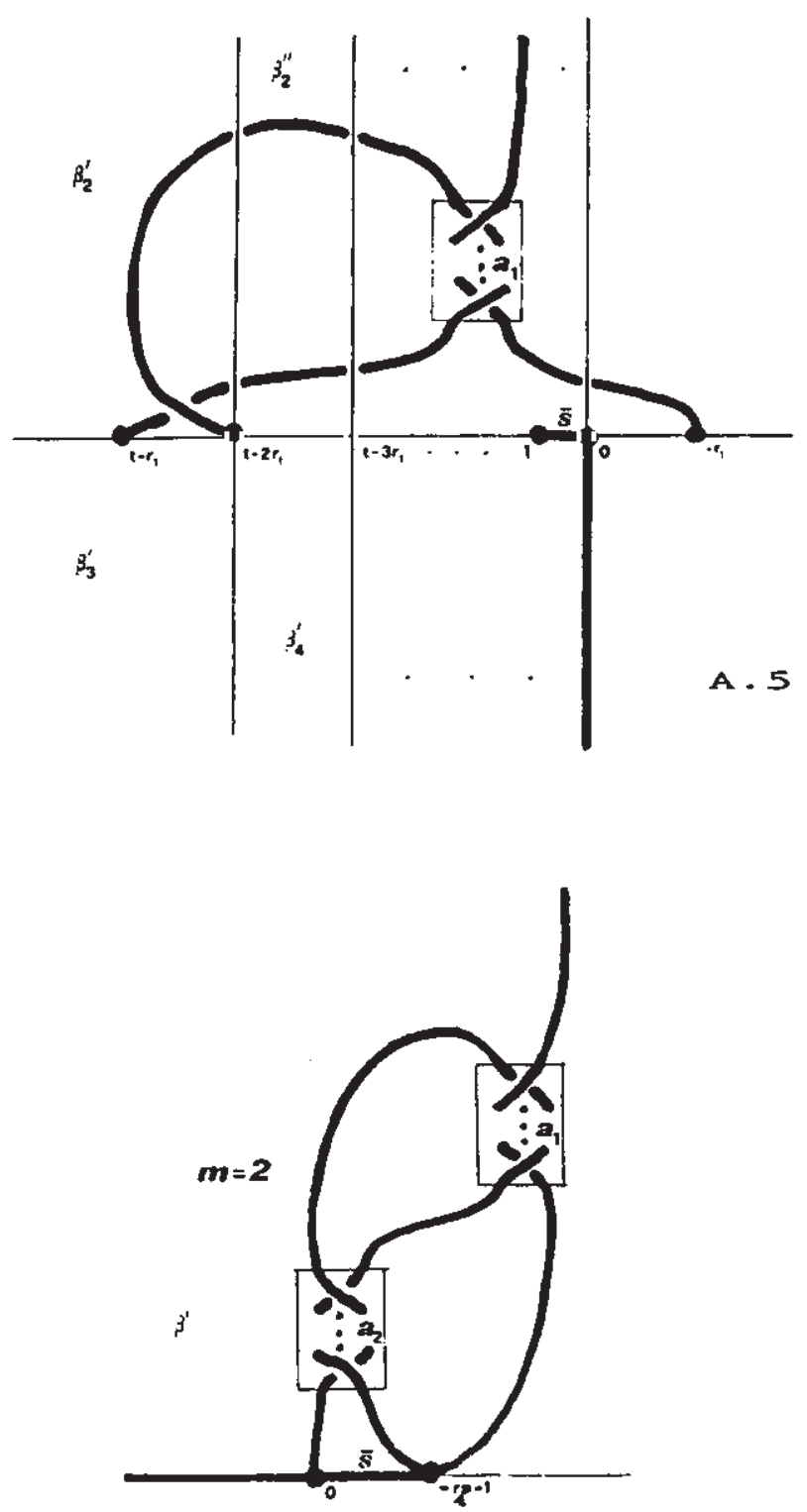

9

A. 6 

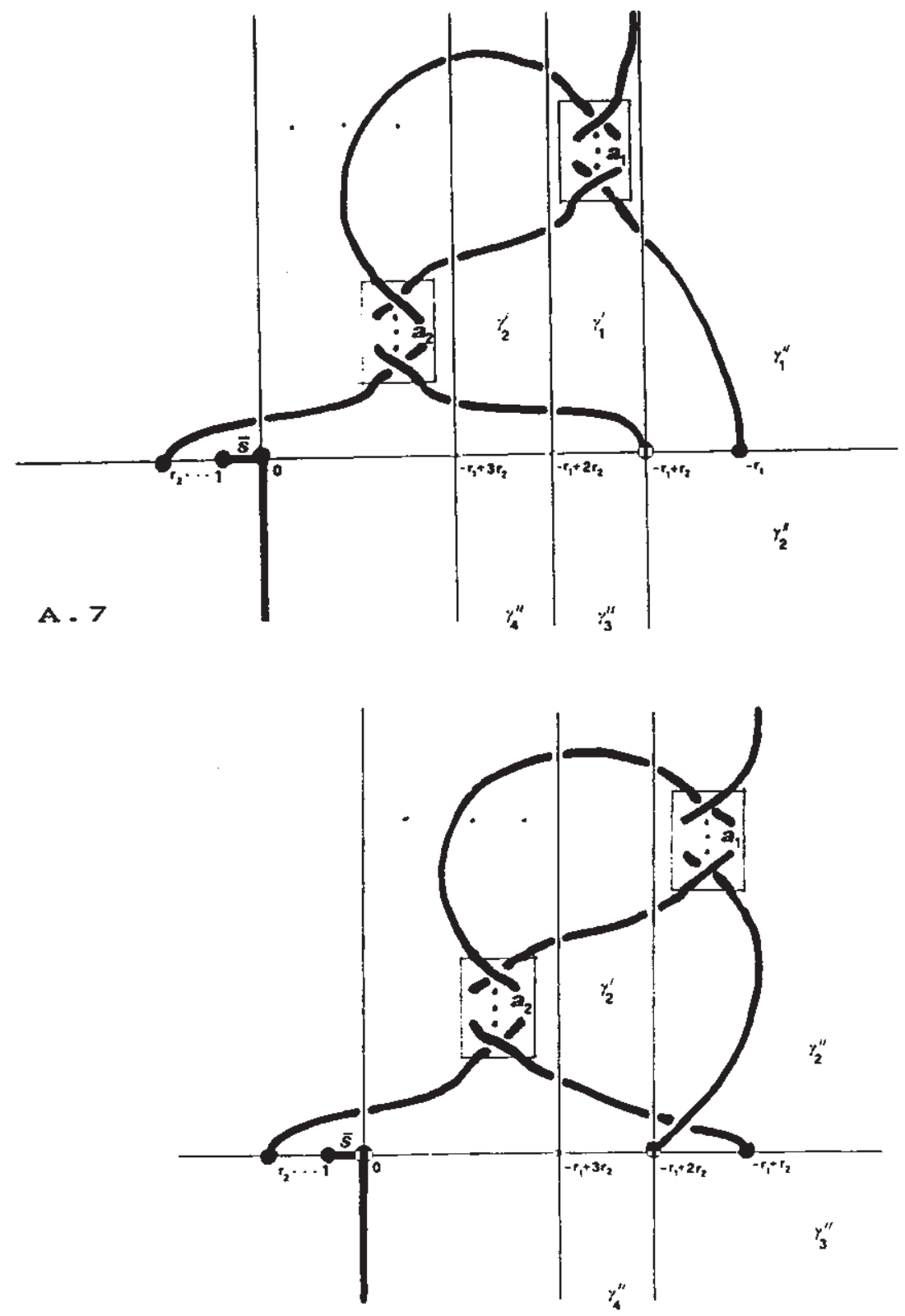

A. 8 


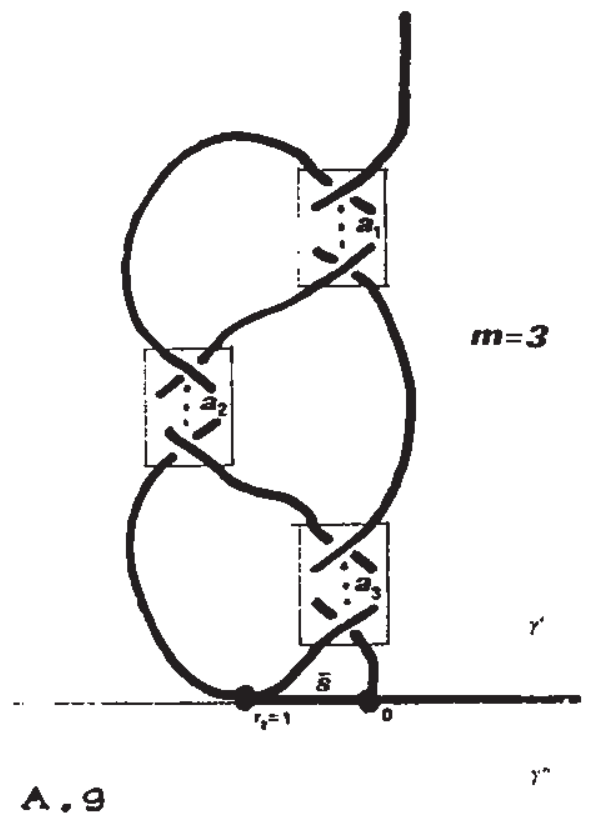

\section{References}

[1] Bernstein, I. and Edmons, A.L., On the construction of branched coverings of low-dimensional manifolds, Trans. Amer. Math. Soc. 247 (1979), 87-124.

[2] Burde, G. and Zieschang, H., Knots, de Gruyter, Berlin-New York, 1985 .

[3] Cavicchioli, A., Pseudo-dissezioni e triangolazioni contratte di spazi con singolarità isolate, Atti Sem. Mat. Fis. Univ. Modena 27 (1978), $132-150$.

[4] Cavicchioli, A., Lins-Mandel crystallizations, Discrete Math. 57 (1985), 17-37. 
[5] Costa, A.F. and Del Val-Melus, P., Crystallizations and permutations representing 3-manifolds, Boll. Un. Mat. Ital. 7, 1-B (1987), 477-490.

[6] Ferri, M., Una rappresentazione delle n-varietà topologiche triangolabili mediante grafi $(n+1)$-colorati, Boll. Un. Mat. Ital. 13-B (1976), 250-260.

[7] Ferri, M. and Gagliardi, C., The only genus zero $n$-manifold is $S^{n}$, Proc. Amer. Math. Soc. 85 (1982), 638-642.

[8] Ferri, M., Gagliardi, C. and Grasselli, L., A graph-theoretical representation of $P L$-manifolds-A survey on crystallizations, Aequationes Math. 31 (1986), 121-141.

[9] Fox, R.H., Covering spaces with singularities, Algebraic Geometry and Topology, a simposium in honour of S.Lefschetz, Princeton Math. Series, vol. 12, 1957, pp. 243-257.

[10] Gagliardi, C., A combinatorial chanacterization of 3-manifold crystallizations, Boll. Un. Mat. Ital. 16-A (1979),441-449.

[11] Gagliardi, C., Extending the concept of genus to dimension n, Proc. Amer. Math. Soc. 81 (81), 473-481.

[12] Grasselli, L., Standard presentations for 3-manifold groups, to appear.

[13] Harary, F., Graph theory, Addison-Wesley, Reading, 1969.

[14] Hilton, P.J. and Wylie, S., An introduction to algebraic topology Homology theory, Cambridge, 1960.

[15] Hudson, J.F.P., Piecewise linear topology, Benjamin, New York, 1969 .

[16] Lins, S. and Mandel, A., Graph-encoded 3-manifolds, Discrete Math. 57 (1985), 261-284.

[17] Mohar, B., Branched coverings, Discrete Comput. Geom. 3 (1988), 339-348.

[18] Montesinos, J.M., Representing 3-manifolds by a universal branching set, Math. Proc. Camb. Phil. Soc. 94 (1983), 108-123. 
[19] Montesinos, J.M., Lectures on branched coverings, Atti del Convegno di Studio sulla Geometria delle varietà differenziabili, I. Cattaneo Gasparini ed., Pitagora Editrice, Bologna, 1985, pp. 127-167.

[20] Mulazzani, M., Lins-Mandel graphs representing 3-manifolds, to appear on Discrete Math..

[21] Mulazzani, M., All Lins-Mandel spaces are branched cyclic coverings of $S^{3}$, to appear on Journal of Knot Theory and its Ramifications.

[22] Neuwirth, L.P., Knot groups, vol. 56, Ann. Math. Studies, 1965.

[23] Pezzana, M., Sulla struttura topologica delle varietà compatte, Atti Sem. Mat. Fis. Univ. Modena 23 (1974), 269-277.

[24] Rourke, C. and Sanderson, B., Introduction to piecewise-linear topology, Springer Verlag, 1972.

[25] Seifert, H. and Threlfall, W., A textbook of topology, Academic Press (English reprint), 1980.

[26] Vecchioli, G., Metodi di rappresentazione di trivarietà chiuse, Tesi di Laurea, Università di Perugia, 1986.

[27] Vince, A., Combinatorial maps, J. Combin. Theory Ser. B 34 (1983), 1-21.

Dipartimento di Matematica,

Universita Di Bologna,

Piazza di Porta San Donato 5,

40127 Bologna, ITALY

E-mail: mulazzadm.unibo.it
Recibido: 13 de mayo, 1994

Revisado: 27 de Febrero, 1995 